\title{
Hydrogel as a Medium for Fluid-Driven Fracture Study
}

\author{
N.J. O'Keeffe ${ }^{1}$ (D) P.F. Linden ${ }^{1}$
}

Received: 12 March 2017 / Accepted: 2 July 2017 / Published online: 1 August 2017

(C) The Author(s) 2017. This article is an open access publication

\begin{abstract}
In this paper we describe how to construct polyacrylamide hydrogels to study the processes linked with hydraulic fracturing. These transparent, linearly elastic and brittle gels permit fracturing at low pressures and speeds allowing accurate measurements to be obtained. In the context of hydraulic fracturing, the broad range of modulus and fracture energy values that are attainable allow experimental exploration of particular regimes of importance. We also describe how material properties may be deduced from hydraulic fracturing experiments. Lastly, we analyse the fracture surface patterns that emerge from fluid-driven cracks occurring within the medium. These patterns are similar to those that have been observed in other materials and we comment on their fractal-like nature.
\end{abstract}

Keywords Hydraulic fracturing $\cdot$ Hydrogels $\cdot$ Fractals

\section{Introduction}

Polyacrylamide hydrogels are widely used as materials in biology as cell culture substrates and for gel electrophoresis to separate proteins. The gel is a highly swollen network

This work was funded with the support of an iCASE award from the EPSRC and BP (Grant No. EP/L505389/1).

N.J. O'Keeffe

okeeffen@damtp.cam.ac.uk

P.F. Linden

p.f.linden@damtp.cam.ac.uk

1 Department of Applied Mathematics and Theoretical Physics, University of Cambridge, Wilberforce Road, Cambridge CB3 0WA, UK of cross-linked acrylamide polymer chains. The material constants, such as elasticity and stiffness, of this gel can be altered by varying the quantity of monomers and crosslinker present. These hydrogels have been approximated as linear elastic materials as they retain a constant storage modulus while under a large range of strains [1].

In the context of fracturing, these gels have been used to study the propagation of dynamic fracture and have been shown to fracture similarly to other brittle materials (e.g. PMMA and glass) [2]. This medium has been used extensively in the study of microbranching and oscillation instabilities involved in the dynamics of two dimensional rapid fracture [3-5].

Fracture dynamics are normally extremely hard to capture due to difficulties in visualising the tip of a crack moving at velocities of the order of the Rayleigh wave speed. One of the main advantages of brittle polyacrylamide gels is that Rayleigh wave speeds are $\sim 5-20 \mathrm{~m} / \mathrm{s}$ are $2-3$ orders of magnitude lower than in 'standard' brittle materials (glass $\sim 3500 \mathrm{~m} / \mathrm{s}$, PMMA $\sim 1600 \mathrm{~m} / \mathrm{s}$ ) [2]. This slows down the fracturing process, allowing accurate measurements to be obtained with the use of a high speed camera.

Recently however, Denisin and Pruitt [6] have reviewed measurements of the mechanical properties of hydrogels, noting that 'reported elastic modulus for the same formulations differ widely' and depend on 'polyacrylamide formulation, ployacrylamide temperature, gelation time and storage duration'. In this paper we will describe the production of hydrogels with consistent and predictable mechanical properties and a novel experimental setup involving brittle, heavily cross-linked polyacrylamide hydrogels used to investigate fluid-driven fracture. This setup will also allow simple measurements of fracture energy and stress intensity for these gels with varying concentrations of monomers and 
cross-linking. Moreover, it provides a platform to examine step-line patterns present on the crack surfaces. Calculating the fractal dimension of these patterns can exhibit similarities with geological samples and other fracture surface phenomena.

\section{Hydraulic Fracturing}

Hydraulic fracturing is a procedure in which a fracture is initiated and propagates due to pressure (hydraulic loading) applied by a fluid introduced inside the fracture. In this study we will focus on a radial or 'penny shaped' fracture (Fig. 1) driven by an incompressible Newtonian fluid, injected at a constant rate into an impermeable gel matrix where we assume there is a negligible fluid lag - i.e. the fluid front coincides with the fracture front.

The technique of hydraulic fracturing is mainly used as a well-stimulation technique in unconventional reservoirs, which have low permeability and porosity making it difficult to extract oil and gas [7]. The creation of fractures in rock formations increases the overall porosity, allowing hydrocarbons to escape. Other applications include measurement of existing (in-situ) stresses [8], carbon sequestration [9], geothermal energy reservoirs [10], compensation grouting [11] and disposal of toxic liquid waste deep underground. This process is also encountered in nature when studying magma transport [12]. In this last case, the mechanism for crack propagation of magma-driven dykes is the pressure caused by density differences between the fluid and surrounding rock formation.

\section{Theory}

We will consider a tensile crack (mode I) where fracturing occurs under the linear elastic fracture mechanics (LEFM) assumption of a propagation criterion [13]:

$K_{I}=K_{I C}$,

where $K_{I}$ is the stress intensity factor and $K_{I C}$ is the fracture toughness related to the material properties.

Viscous dissipation within the fracture can play a significant role in the shape and speed of the fracture tip. The

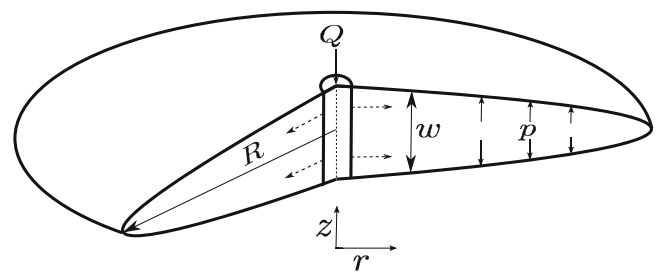

Fig. 1 Schematic diagram showing the radial fracture geometry dominance of viscous dissipation relative to energy dissipation from bond-breaking due to the toughness of the material can be related by a characteristic time. This gives the time in which a fracture transitions from a viscously dominated propagation regime to a material toughness dominated regime [14]:

$t_{m k}=\left(\frac{\mu^{5} Q^{3} E^{\prime 13}}{K_{I C}{ }^{18}}\right)^{1 / 2}$,

where $\mu$ is the dynamic viscosity, $Q$ is the volumetric injection rate, and $E^{\prime}=E /\left(1-v^{2}\right)$ is the plane strain modulus relating Young's modulus $E$ and Poisson ratio $v$.

When the fracture propagates in the toughness dominated regime the crack tip thickness $w$ can be approximated using the LEFM asymptote [15]:

$w \sim\left(\frac{32}{\pi}\right) \frac{K_{I}}{E^{\prime}} x^{1 / 2}, \quad \frac{x}{R} \ll 1$,

where $x$ is the distance from the crack tip and $R$ is the fracture radius.

\section{Hydrogel}

\section{Gelation of Polyacrylamide Hydrogels}

The hydrogels were prepared by free radical polymerization of acrylamide (Sigma Aldrich) as the monomer and $\mathrm{N}, \mathrm{N}^{\prime}$-methylenebis (acrylamide) (Sigma Aldrich) as the cross-linker in aqueous solution. This means any oxygen present in the solution will inhibit the polymerization. The initiator ammonium persulphate (APS) and accelerator $\mathrm{N}, \mathrm{N}, \mathrm{N}, \mathrm{N}^{\prime}$-tetramethylethylenediamine (TEMED) (Sigma Aldrich) then catalyze the reaction. The gels contain differing amounts of acrylamide produced from a $40 \% \mathrm{w} / \mathrm{v}$ (weight/volume) stock solution and bis-acrylamide in solid form, that are added to a certain amount of demineralized water to give the desired $\% \mathrm{w} / \mathrm{v}$ of each chemical. This solution is then degassed for 10-15 minutes to counteract oxygen inhibiton of the reaction, which is crucial for reproducibility of the gel. Degassing is done by placing the solution inside a vacuum chamber which is connected to a pump. A low pressure environment is then created causing air to be removed from the liquid. Next, $0.075 \%$ w/v of APS was added and finally $0.05 \% \mathrm{v} / \mathrm{v}$ of TEMED (all volume percentages are of the final volume).

We constructed gels of dimensions $100 \times 100 \times 77 \mathrm{~mm}$ which is equivalent to $770 \mathrm{~mL}$ of volume. The total percentage of acylamide monomer and bis-acrylamide cross-linker varied from $8-15 \% \mathrm{w} / \mathrm{v}$ and $2.5-6 \% \mathrm{wt}$. (cross-linker weight/monomer weight). Each gel was prepared at room temperature $\left(20{ }^{\circ} \mathrm{C}\right)$ and the solution was left for over 2 hours allowing gelation to occur fully. During this gelation 
period the chamber containing the solution is covered with parafilm tape, as an additional precaution against oxygen inhibiting the polymerization.

\section{Properties}

Polyacrylamide gels are recognised as linearly elastic materials in the literature $[16,17]$. These gels consist of crosslinked polymer chains. Their elastic properties are determined by the concentration of monomers, acrylamide, and cross-linking molecules, bis-acrylamide. An increase in the monomer \% w/v will result in an increase in the Young's modulus and fracture energy, if the cross-linking \% wt. is held constant. Furthermore, increasing the cross-linking $\%$ wt. will make the gel more brittle and decrease the fracture surface energy. This complicated relationship means that only a few measurements have been found in the literature for fracture energies at particular gel percentages $[18,19]$. Normally these fracture energy values are obtained from peel tests. Here, we approached the measurement of these values using a technique outlined in "Determination of Stress Intensity and Fracture Surface Energy". It has been shown that an increase in monomer will always lead to an increase in Young's modulus [6]. However, an increase in cross-linker will also increase the gel modulus up to around $7 \%$ wt. cross-linking. If the cross-linking is increased beyond this, the gels become heterogeneous and the value of the Young's modulus will plateau.

Heavily cross-linked polyacrylamide gels are transparent, elastic and brittle materials. Elasticity and brittleness are two different properties. However, they are not independent for elastic solids. Ideally, fracture toughness $K_{I C}$ follows the relation:

$K_{I C}=\sqrt{2 \gamma_{s} E^{\prime}}$

where $\gamma_{s}$ is the fracture surface energy, which can be defined as the energy required to create one unit of surface area. Equation (4) incorporates both elasticity and brittleness properties in the Young's modulus and fracture surface energy, respectively.

Rate-dependent fracture surface energies $\gamma_{s}$ have been observed in several gel systems. Chemically cross-linked polyacrylamide hydrogels exhibit a rate dependence that decreases with increasing density of chemical cross-links [19]. Since we are using heavily cross-linked hydrogels we will assume the fracture energy to be constant which can be determined from the measurements obtained in "Determination of Stress Intensity and Fracture Surface Energy". This is an advantage over gelatin systems, where the fracture energy varies linearly with crack velocity and the rate sensitivity increases with the amount of physical cross-linking [20].
The permeability of these gels can be extremely low and comparable to that observed in unconventional hydrocarbon reservoirs. In shale gas formations the expected permeability is in the range $10^{-6}-10^{-8}$ Darcy, while in polyacrylamide hydrogels permeability values can range from $10^{-7}-10^{-8}$ Darcy [21].

\section{Experimental Setup}

The experimental setup consists of setting a hydrogel of dimensions $100 \times 100 \times 77 \mathrm{~mm}$ around an injection needle of radius $0.81 \mathrm{~mm}$ as depicted in Fig. 2. A large gel matrix is used so that the free surface and boundaries have a negligible effect on the stress state near the injection point, at the bottom of the needle. To study the idealised model we inject fluid at a constant volumetric rate, ranging from $10-23 \mathrm{~mL} / \mathrm{min}$, into the hydrogel using a syringe pump (WPI AL6000).

We require the fracture to propagate radially and perpendicular to the injection needle, and use a high speed camera (Dalsa Falcon 2 4MP 120fps) to aid visualisation. To achieve this we must orientate the needle parallel to the minimum confining stress, as the fracture will propagate perpendicular to this direction. This is due to the fact that the radial crack is a tensile fracture (mode I) and opens in the direction of least resistance. Thus, four rectangular plates of width $1 \mathrm{~mm}$ are inserted parallel to the needle on each side of the gel as seen in Fig. 2(b). These plates are made from polycarbonate which were chosen due to their transparency, strength and re-usability. This also places the gel in an approximate condition of plane strain, as all the stresses are acting perpendicular to the injection needle. The incompressible Newtonian fracturing fluids used include water, glycerin, silicone and golden syrup, with viscosities ranging from $\mu \sim 10^{-3}-10^{1} \mathrm{~Pa} \cdot \mathrm{s}$.

The propagation of these fluid fractures was clearly observed due to the transparent nature of the gels. The radial fracture profile is found by dyeing the fluid, so that it can be easily distinguished from the surrounding medium. The Digiflow software was used extensively in processing the videos and taking measurements [22]. The resolution we are able to achieve with these measurements is 1 pixel $\approx 0.04$ $\mathrm{mm}$.

\section{Thickness Measurement}

Dye attenuation was used to measure fracture width. This is a process in which the absorbance of light is used to correspond to thickness measurements. To do this a red LED light sheet with a diffuser was used as background lighting to provide a uniform monochromatic light source. The injected fluid is dyed with methylene blue, which strongly absorbs 
Fig. 2 Schematic diagrams of the experimental setup

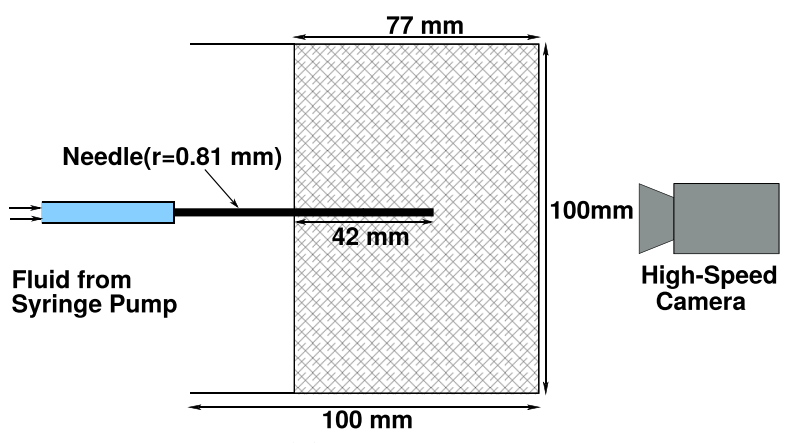

(a) Elevation view

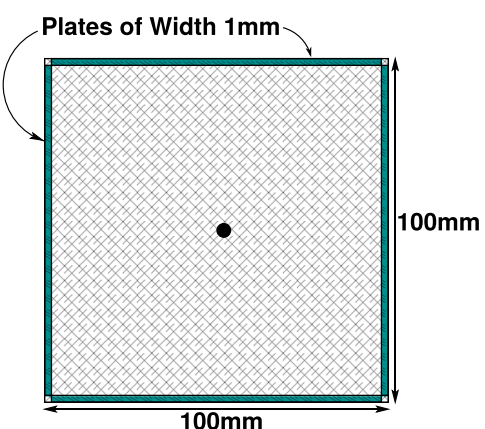

(b) Plan view at the wavelength of the red light source. The absorption of this background light is then directly related to the amount of fluid through which it passes.

\section{Calibration}

To correlate intensity values across the dyed fluid region with crack thickness, we perform a calibration experiment with the same fracturing fluid using a glass wedge with a linearly increasing thickness from $0-8 \mathrm{~mm}$ filled with the dyed fracturing fluid. The wedge was placed in our acrylic container (Fig. 2) and a polyacrylamide gel formed around it, in order to take into account the absorption of light by the gel matrix itself. We denote the intensity of the uniform background light that has travelled through the polyacrylamide gel only by $I_{0}$, and the intensity distribution of light that passed through the fluid filled region by $I$. Normalising the fluid-filled light intensity with the background intensity through the gel, the absorption of the light due to the matrix alone can be excluded. We plot this normalisation $I / I_{0}$ versus the thickness of the wedge in Fig. 3(b). A polynomial fit to the calibration data was then calculated and used to relate the light intensity data to thickness measurements for fluid filled fractures, under the assumption that the fracture is symmetric about its mid plane.

\section{Results}

\section{Young's Modulus}

We have conducted a series of compression tests on sample rectangular gels to obtain material properties, such as the Poisson's ratio, and observe the variability when concentrations are altered. This was done on an Instron 3345, using parallel plates to measure compressive stress and strain. A sample stress-strain graph can be seen in Fig. 4(c) for a 13 $4 \%$ gel. These tests produced an approximate Poisson's ratio $v \approx 0.48$, which agrees with the literature that the hydrogels can be treated as linearly elastic [23].

Using the principle of determination of Young's modulus by Hertzian contact, or indentation with a solid sphere [24, 25], we calculated a range of elastic moduli for different monomer to cross-linker ratios. This method was carried out using a TA.XT Texture Analyser (Stable Micro Systems). A sphere of radius $a=0.63 \mathrm{~cm}$ is indented a certain distance

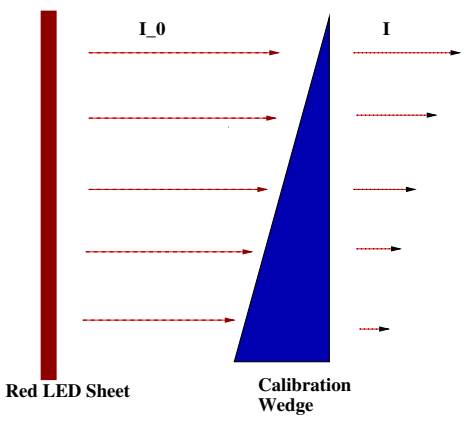

(a) Calibration

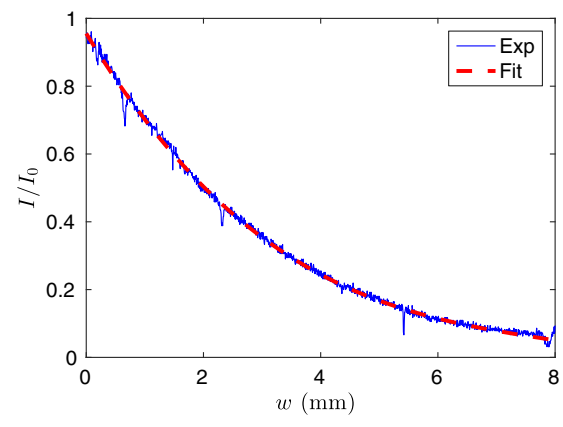

(b) Intensity vs thickness

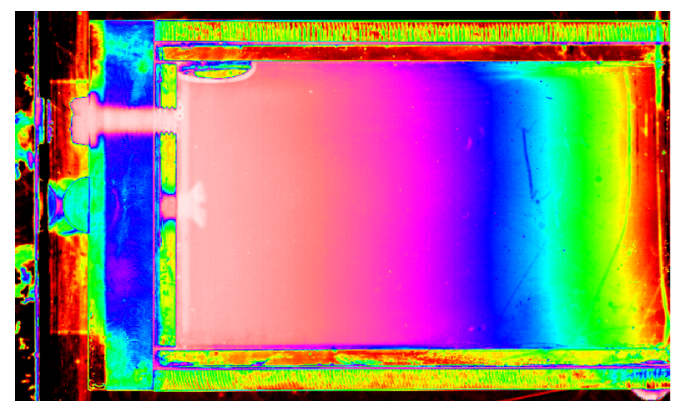

(c) Experimental calibration

Fig. 3 (a) A red monochromatic light of uniform intensity $I_{0}$ is obtained from an LED light sheet and diffuser. This light then passes through the hydrogel and calibration wedge or fluid fracture with blue dye and emerges at a diminished intensity $I$ depending on the path length through the fluid. (b) The normalised light intensity $I / I_{0}$ versus the corresponding thickness of the fluid filled region for a given dye concentration. (c) Experimental image of a calibration using glycerin with $\mu=1130 \mathrm{mPa} . \mathrm{s}$ and a concentration of Methylene blue of $0.05 \mathrm{~g} / \mathrm{L}$ 
Fig. 4 Characterisation of the gel Young's modulus. (a) Experimental technique of Hertzian contact using spherical indentation. (b) Force versus $\delta^{3 / 2}$ for a 13 - $6 \%$ gel. (c) Sample stress-strain curve from a compression test of a $13-4 \%$ gel. (d), (e) Young's modulus versus monomer $\% \mathrm{w} / \mathrm{v}$ for fixed cross-linking of $2.5 \% \mathrm{wt}$. ( $m=22.5, c=-121.5)$ and $6 \%$ wt. ( $m=47.5, c=-316.7)$, respectively. (f) Young's modulus versus cross-linking $\%$ wt. for fixed monomer concentration of $10 \% \mathrm{w} / \mathrm{v}$ $(m=16.6, c=59.1)$

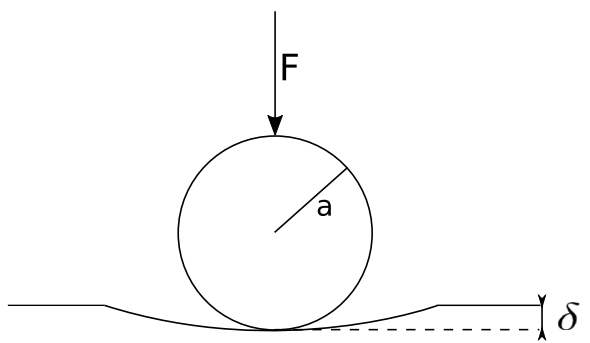

(a) Hertzian contact

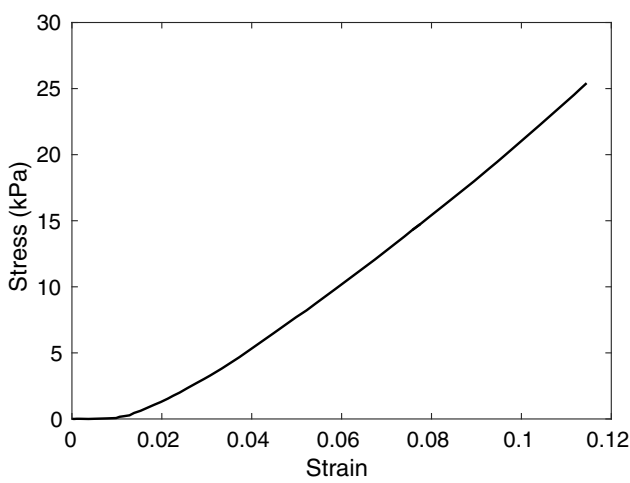

(c) Stress-strain

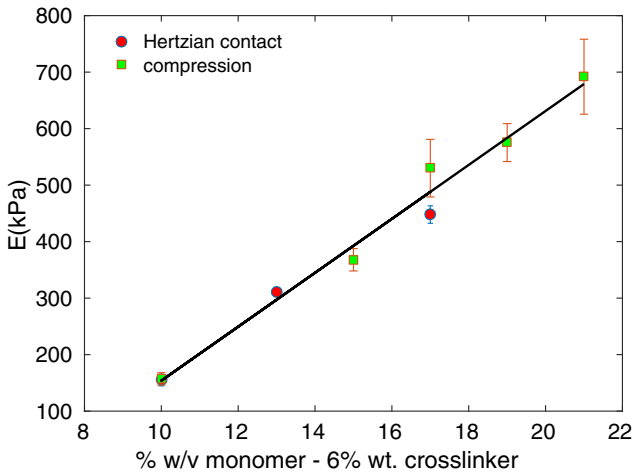

(e) $6 \%$ wt. cross-linker

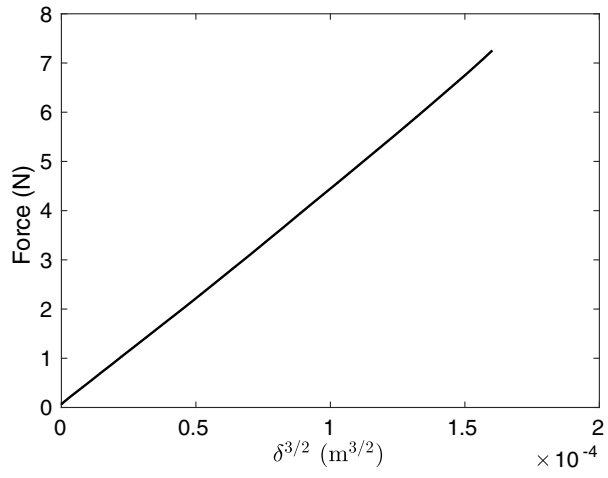

(b) F vs. $\delta^{3 / 2}$

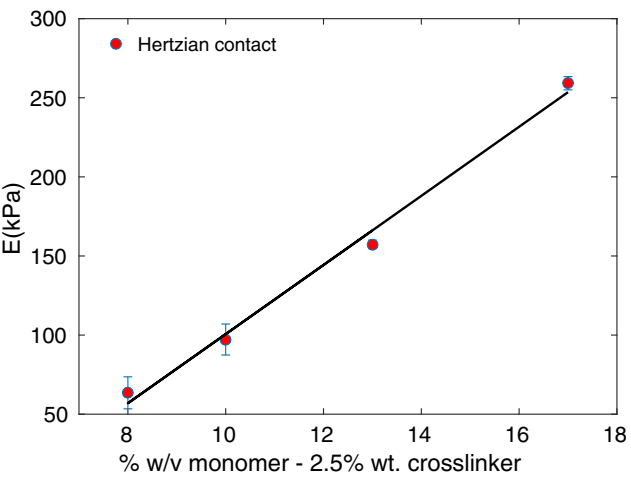

(d) $2.5 \%$ wt. cross-linker

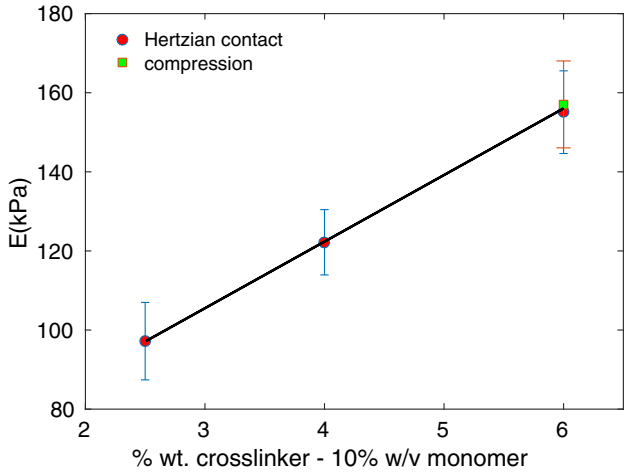

(f) $10 \% \mathrm{w} / \mathrm{v}$ monomer onto the surface of the hydrogel as seen in Fig. 4(a) and the force measured. For a perfectly elastic solid, if the surface is displaced a distance $\delta$ under a force $F$, the plane strain modulus $E^{\prime}$ is given by the relation:

$E^{\prime}=\frac{E}{1-v^{2}}=\frac{3}{4 a^{1 / 2}} \frac{F}{\delta^{3 / 2}}$.

However to exclude surface tension effects in the initial stages of indentation, the slope of the linear section of a $\delta^{3 / 2}$ versus $F$ plot, an example of which can be seen in Fig. 4(b), is used to calculate the Young's modulus $E$ :

$E=\frac{3\left(1-v^{2}\right)}{4 a^{1 / 2}} \frac{\Delta F}{\Delta \delta^{3 / 2}}$.
The results of these tests can be seen in Fig. 4 and Table 1. We conducted two indentation experiments at different places on the gel surface for each sample. The maximum deviation from the average value was always less than $2.5 \%$. The error bars are estimated from measurement uncertainty on the slope calculation. From the figures we can clearly see that increases in the amount of monomer and cross-linker, in the ranges shown, both contribute to an increase in Young's modulus. From Fig. 4 it is evident that monomer percentage has a larger effect on the elastic modulus than cross-linking. The linear fits seen in Fig. 4(d), (e) and (f) have a slope $m$ and intercept $c$. It has been shown that higher percentages of cross-linking can lead to a plateau in the elastic modulus values and the relationship is more complex [6]. 
Table 1 Young's modulus, stress intensity and fracture energy measurements

\begin{tabular}{llll}
\hline $\begin{array}{l}\text { Gel concentration } \\
\% \mathrm{w} / \mathrm{v}-\% \mathrm{wt.}\end{array}$ & $\begin{array}{l}\text { Young's modulus } \\
E(\mathrm{kPa})\end{array}$ & $\begin{array}{l}\text { Stress intensity } \\
K_{I}\left(\mathrm{~Pa} \mathrm{~m}^{1 / 2}\right)\end{array}$ & $\begin{array}{l}\text { Fracture energy } \\
\gamma_{s}\left(\mathrm{~J} \mathrm{~m}^{-2}\right)\end{array}$ \\
\hline $8-2.5$ & $64 \pm 8$ & $460 \pm 27$ & $1.2 \pm 0.2$ \\
$10-2.5$ & $97 \pm 9$ & $631 \pm 36$ & $1.4 \pm 0.2$ \\
$10-6$ & $155 \pm 10$ & $623 \pm 17$ & $0.9 \pm 0.05$ \\
$13-2.5$ & $157 \pm 3$ & $738 \pm 56$ & $1.3 \pm 0.2$ \\
$13-6$ & $311 \pm 7$ & $845 \pm 77$ & $0.86 \pm 0.16$ \\
$15-6$ & $367 \pm 20$ & $939 \pm 65$ & $0.9 \pm 0.1$ \\
\hline
\end{tabular}

Experimentally we can obtain a wide range of values for the Young's modulus, with values varying from at least $50-700 \mathrm{kPa}$. This was one of our main motivations for conducting experiments in this medium, as it allowed us considerable variation in the transition time (equation (2)) derived for hydraulic fractures.

\section{Determination of Stress Intensity and Fracture Surface Energy}

We can use our measurements of fracture thickness, cracktip behaviour and radial extent to consider the stress intensity factor [26]. This can be done in the toughness limiting regime where the crack tip LEFM asymptote (equation (3)) relates the fracture thickness, distance from the tip and stress intensity. We consider only mode I opening where we can use the stress intensity factor $K_{I}$ to estimate the fracture toughness $K_{I C}$ of the hydrogel matrix, under the LEFM assumption that propagation occurs when (equation (1)) is satisfied.

Plotting the opening over $60 \%$ of the fracture radius normalised according to equation (3) produces Fig. 5, where the stress intensity factor $K_{I}$ is the value averaged over the crack tip region. The thickness is plotted for 10 time steps with the black line representing the average of these

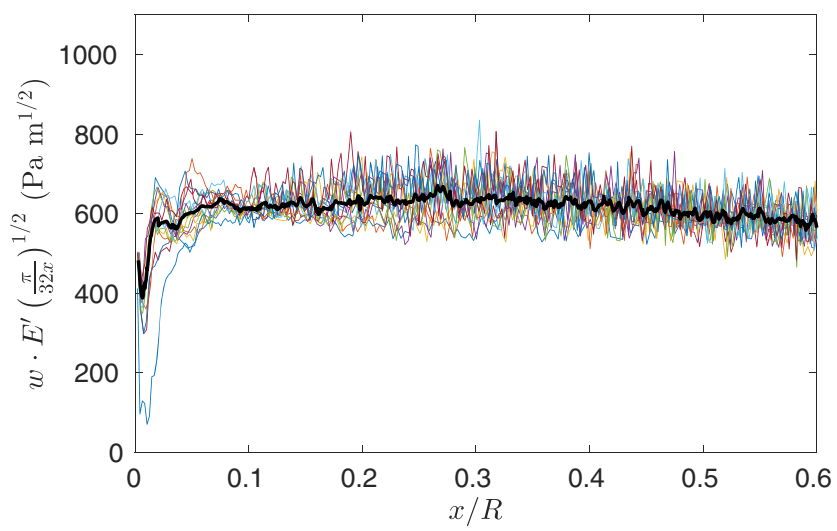

Fig. 5 Fracture opening normalised by equation (3) versus distance from the crack tip $x / R$. Curves are plotted at 10 different time steps with the black line indicating the average. The stress intensity factor $K_{I}$ is given by the limit as $x / R \rightarrow 0$ experimental curves. This particular experiment corresponds to a gel concentration of $10 \% \mathrm{w} / \mathrm{v}$ monomer and $2.5 \%$ wt. cross-linker. From the propagation criterion we assume $K_{I}=K_{I C}$ and we can deduce that $K_{I C}=631 \pm 36$ $\mathrm{Pa} \mathrm{m}^{1 / 2}$. Thus, we can use equation (4) to estimate the fracture surface energy of this particular gel configuration to be $\gamma_{s} \approx 1.4 \mathrm{~J} / \mathrm{m}^{2}$. The noise near $x / R=0$ is due to the minuscule thickness near the crack tip. The dye attenuation value is extremely small in this area and is comparable to the noise in the experiment due to the limitations of our calibration. However, the data clearly follow a specific trend before the noise becomes significant. The observed constant stress intensity value observed all along the fracture tip, retrieved from using LEFM, suggests that the assumption of a homogeneous brittle material is valid.

This technique was then applied to a number of gel concentrations, the results of which can be seen in Table 1 and Fig. 6. In Fig. 6(a) we can see that the stress intensity value increases with monomer percentage as expected. $K_{I}$ is similar for both 2.5 and $6 \% \mathrm{wt}$. cross-linking, with $2.5 \%$ wt. having marginally higher values.

The similar behaviour in stress intensity values means that the fracture energies for the higher cross-linked gels, calculated using equation (4), will be lower due to larger moduli measurements. The values of fracture energy $\gamma_{s}$ can be seen in Table 1, which are similar to previous values reported from peel testing [18]. In Fig. 6(b), the fracture energy $\gamma_{s}$ for $2.5 \%$ wt. cross-linking varies with larger amounts of monomer. However, at $6 \%$ wt. crosslinking the fracture energy settles to a cross-linking value of $\gamma_{s} \sim 0.9 \mathrm{~J} \mathrm{~m}^{-2}$. This is an extremely useful property of these gels for our hydraulic fracturing experiments, allowing us to change the Young's modulus independent of fracture energy. It allows us to tune the characteristic time for transition (equation (2)) appropriately, so that the viscosity dominated regime may be explored. Otherwise, this would be very difficult experimentally, as we would need to increase both the injection rate $Q$ and fluid viscosity $\mu$ significantly in order to analyse the same parameter space.

Moreover, this technique of determining fracture energy can become extremely useful when trying to characterise heterogeneous or layered gel systems. 
Fig. 6 a Stress intensity $K_{I}$ versus $\% \mathrm{w} / \mathrm{v}$ monomer. $\mathbf{b}$ Fracture energy $\gamma_{s}$ versus $\% \mathrm{w} / \mathrm{v}$ monomer. Both plots refer to gels with 2.5 and $6 \%$ wt. cross-linking

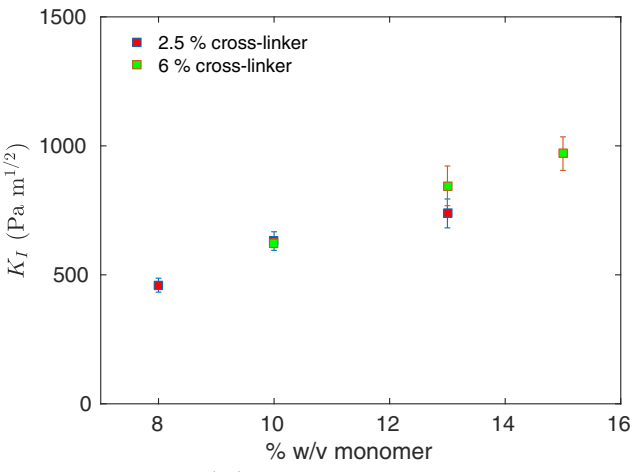

(a) Stress intensity

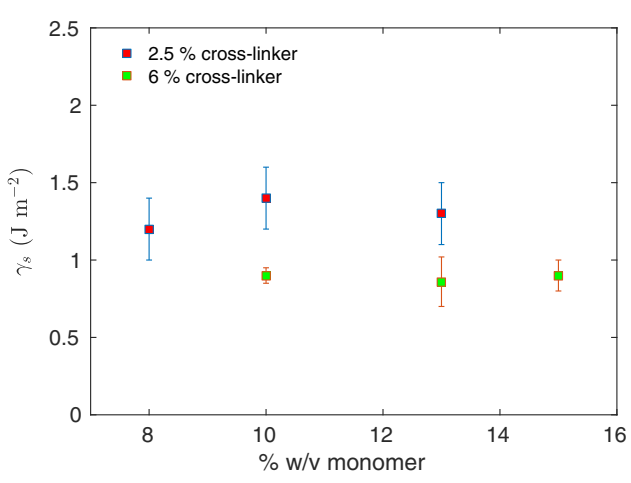

(b) Fracture energy

\section{Fracture Surface}

When fracturing occurs in these hydrogels and the crack surface is examined, varying amounts of roughness are evident. These patterns are similar in shape to shear banding (or strain localization). A shear band is a narrow zone of plastic failure caused by intense strain on the material and corresponds to a loss of homogeneity in the deformation of a material. The phenomenon is most often observed in ductile materials, but is also seen in quasi-brittle materials, e.g. rock, ice and concrete.
However, our hydrogels are brittle materials and the two surfaces post-fracture are identical suggesting that no plastic deformation of the gel matrix has occurred. The patterns evident on the crack surfaces are commonly referred to as 'steps-lines'. These step-lines are small changes in height $\left(\mathcal{O}\left(10^{-1} \mathrm{~mm}\right)\right)$ and have been observed in hydrogels fractured in a peel-test configuration [19, 27]. The step-lines are observed in two forms: orientated at an angle to the crack propagation direction and parallel to the direction of growth. Previously, these patterns have been labelled 'scale' and 'river' step-lines, respectively [27]. Wallner [28]
Fig. 7 Step-line pattern as a result of hydraulic radial fracture in gels with cross-linking between $1.9-2.5 \%$

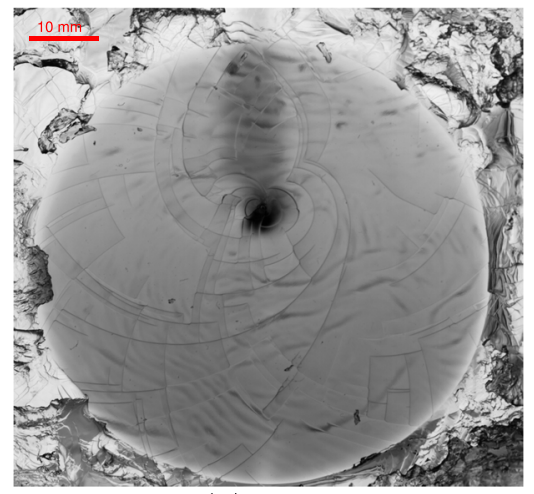

(a) $8-2.5 \%$

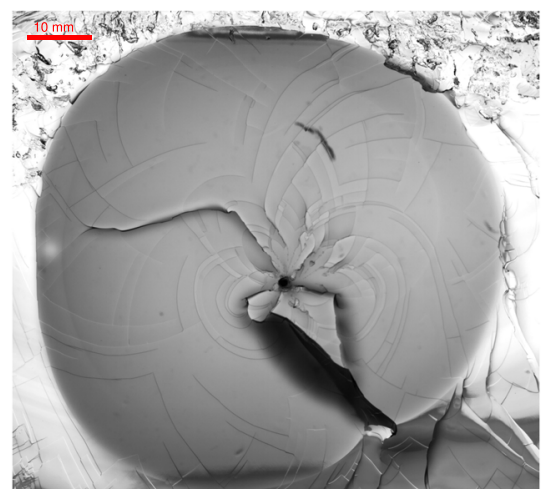

(c) $13-1.9 \%$

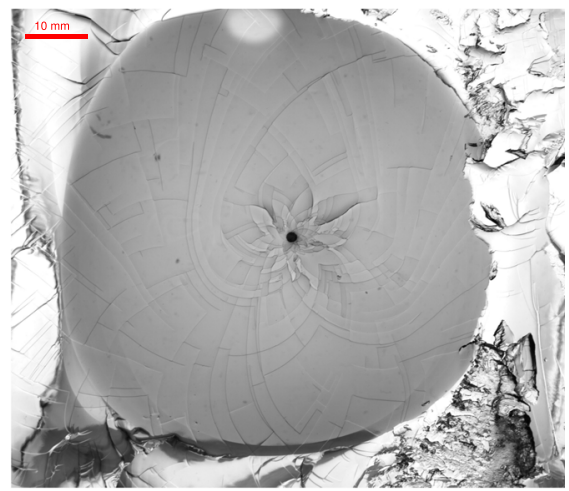

(b) $15-2.5 \%$

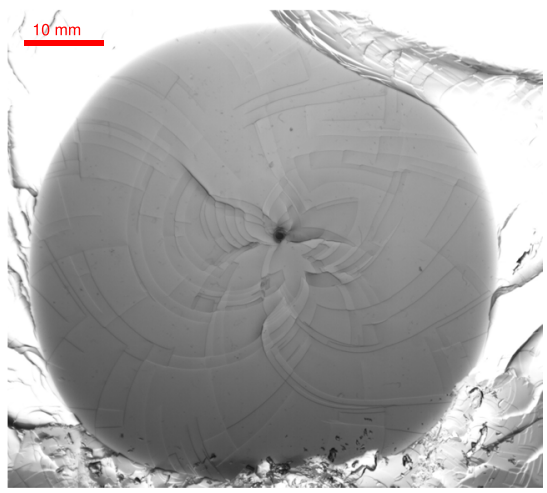

(d) $13-2.5 \%$ 
observed similar step-lines on the crack surfaces of glass. These Wallner lines are explained by proposing that they are the intersections of the paths between the moving crack front and the stress pulses. This mechanism is inadequate for the explanation in these experiments as the fracture velocity is of order $\mathcal{O}\left(10^{-2} \mathrm{~m} / \mathrm{s}\right)$, while the sound velocity is of the order $\mathcal{O}\left(10^{0} \mathrm{~m} / \mathrm{s}\right)$; meaning the intersections would be near the crack tip and approximately perpendicular to the propagation direction.

In Figs. 7 and 8 examples of scale step-line patterns postfracture can be observed. These patterns are created as small areas of the crack tip are aligned towards the fracture propagation direction. The behaviour of these sections is similar to a mode III tearing crack. It has been suggested that in the scale case these regions propagate at an angle of $\pi / 4$ to the propagation direction and in a river pattern they propagate parallel to the propagation direction [27]. The observation of these scale and river step-lines have been found to be dependent on the velocity at which the fracture propagates. As the velocity of the fracture increases, the pattern transitions from a scale to river step-line morphology.

As mentioned, this pattern formation is very similar to shear banding, which has been shown to depend upon the dimensionless parameter [29]:

$B=\frac{p_{c} c}{G V_{b c}}$, where $p_{c}$ is the confining pressure, $c$ is the velocity of sound in the material, $G$ is the shear modulus (or Lamé constant) and $V_{b c}$ is the velocity of the boundary or crack tip. With increasing $B$ the shear bands are located further apart. A common explanation for this is that as a shear band forms, the stress inside the band decreases because of elastic unloading. Outside the shear band the pressure increases, suppressing another band formation in close proximity. The pressure difference then travels through the material at the speed of sound. Therefore, when $B$ is large the banding pattern will occur on a larger scale. On the other hand, when $B$ is small, the sound velocity is smaller compared with the loading rate of the crack and the bands are located closer together in order to release the strain.

Therefore, we know an inverse relationship between spacing and velocity exists in the shear banding case. This relation is also observed for our brittle step-lines. The spacing between patterns increases as the crack tip velocity decreases. This is seen in Fig. 7, as the nucleation of these mode III regions is less evident away from the injection source. Fluid is pumped at a constant rate from the source and the flow rate decreases like $1 / 2 \pi R$ away from this. This leads to a decreasing crack velocity, as the crack propagates radially outwards and any patterns observed further away from this source materialised at a lower velocity.

The formation of these mode III-like zones in our material could be caused by local material inhomogeneities that
Fig. 8 Step-line pattern as a result of hydraulic radial fracture in highly cross-linked gels $(6 \%)$

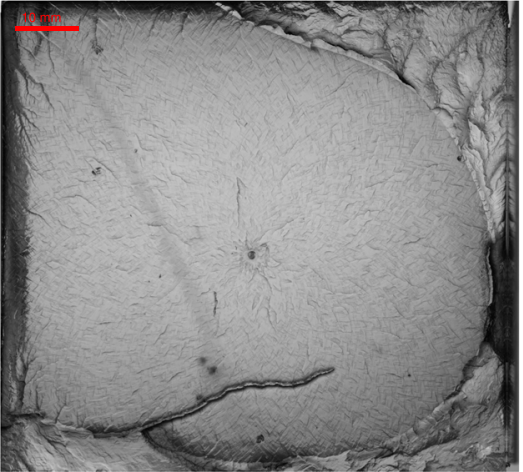

(a) $13-6 \%$

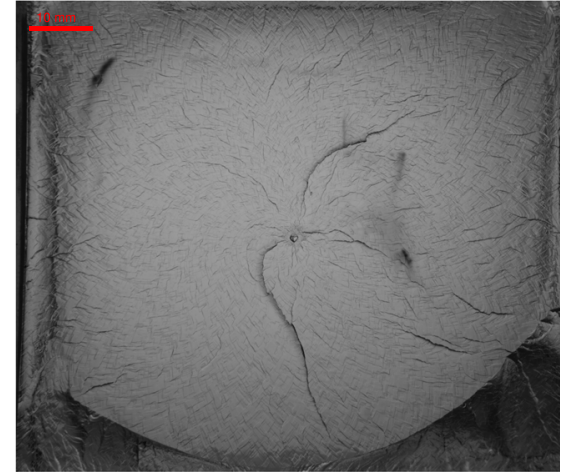

(b) $13.3-6 \%$

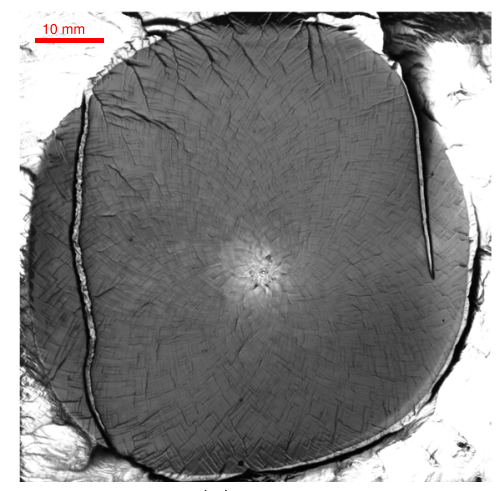

(c) $15-6 \%$ 


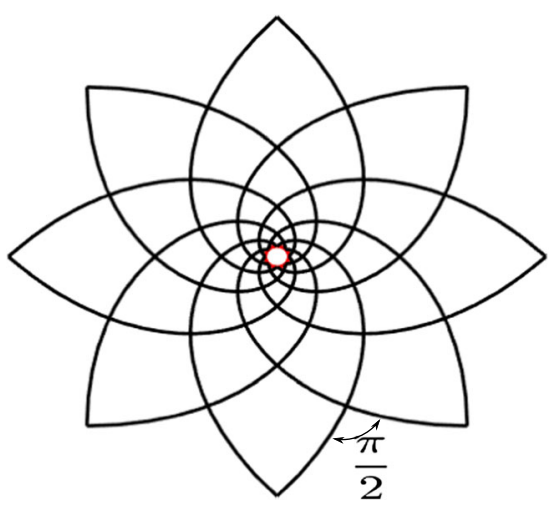

Fig. 9 Theoretical prediction of step-line patterns around a circular indentation with logarithmic spirals pitched at an angle of $\pi / 4$

would cause the crack front to break into numerous sections which propagate at different heights. This may also be due to blunting of the crack tip due to the stresses involved and the existence of small cavities in front of the crack tip. The tensile stress can lead to coalescence of the crack tip and cavities, which may cause the creation of these step-line discontinuities. At lower velocities this softening and cavitation may take longer to occur, due to the crack loading and resulting in fewer discontinuities. Similar corrugations have been observed in other brittle materials such as brittle metallic glasses (BMGs) and glassy polymers [30-32].

As mentioned before, the spacing between patterns has an inverse relation to fracture velocity. The fracture spacing also depends on the material properties of the gel such as monomer and cross-linking percentage which is clear from Figs. 7 and 8. It is expected that the sound velocity of the material increases with increasing cross-linking, due to the larger number of bonds present. Thus, more chains on the fracture surface must be broken in order for the crack to propagate. This higher cross-link density explains why more patterns are seen on the fracture surface in Fig. 8, as any softening of the crack tip and cavitation will occur on a much smaller scale meaning step-lines are located closer together.

If we consider the radial geometry of our fracture and take into account that previous studies have found that these patterns occur at angles of $\pm \pi / 4$ to the direction of propagation [19], we can theoretically predict the shape they will take. Logarithmic spirals pitched at $\pi / 4$ capture this behaviour as seen in Fig. 9. This pattern is extremely similar to those observed in ductile systems through indentation $[33,34]$.

Considering Fig. 10, we measured the angles between step-lines of a specific $15-2.5 \%$ gel post hydraulic fracture. In this example, the angles are consistently less than $\sim 90^{\circ}$ and vary from $\sim 80-90^{\circ}$.

\section{Fractal Analysis}

The step-line patterns on the fracture surfaces also form fractal networks. These beautiful fractal patterns originate from the distribution of these crack surface markings. The fractal dimension of these patterns was computed using the classical box-counting technique [35]. The results of this analysis can be seen in Fig. 11. It is clear from this graph that gels with a cross-linking of $1.9-2.5 \%$ and those with cross-linking $\sim 6 \%$ follow two different curves, in relation to the fractal dimension at specific box sizes. The fractal dimensions are $d f \sim 1.624 \pm 0.143$ and $1.89 \pm 0.08$ for
Fig. 10 Measurement of the angles between emanating step-lines

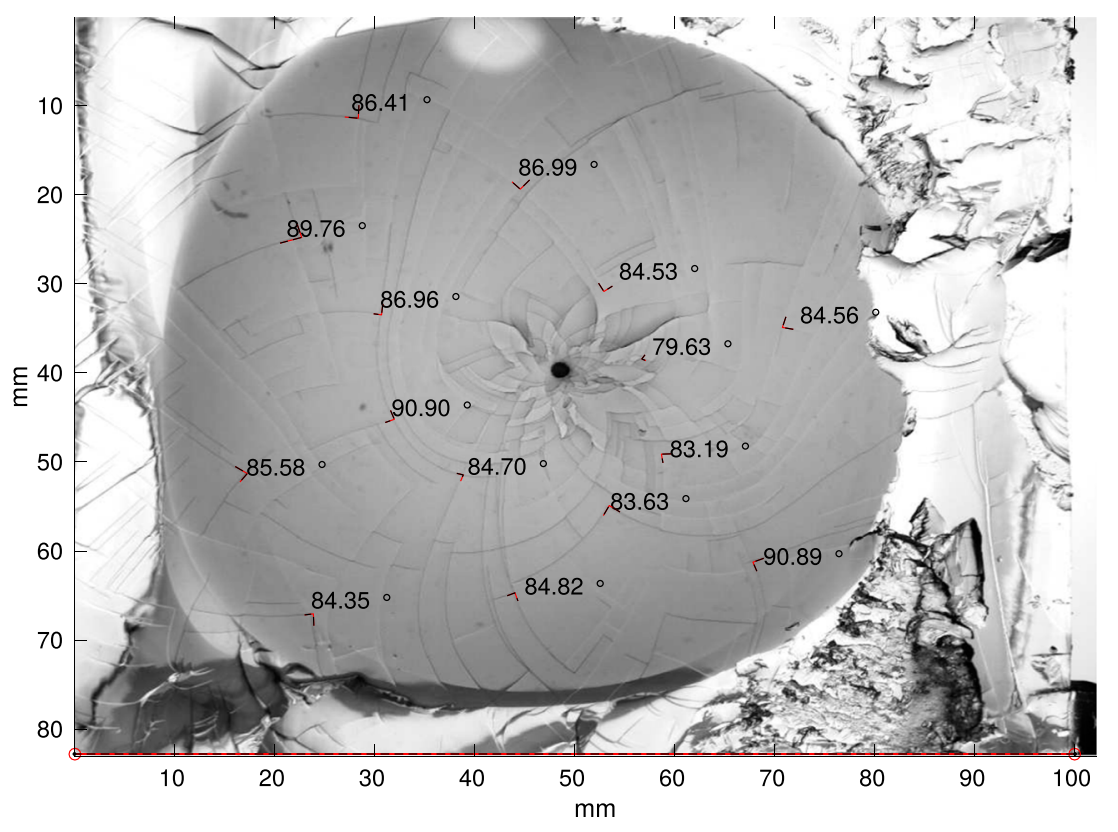




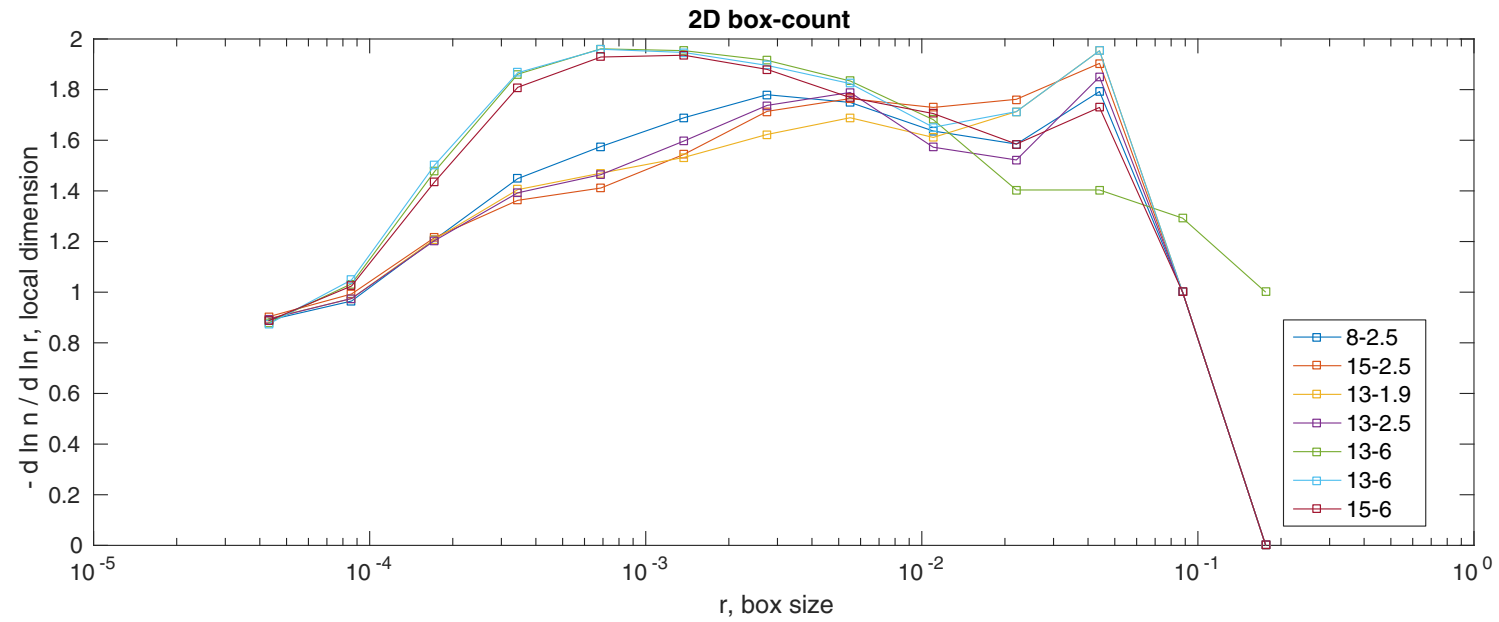

Fig. 11 The fractal dimension analysis using the 'box counting' method for varying gel-concentrations

$1.9-2.5 \%$ and $\sim 6 \%$ cross-linking, respectively. The fractal dimension for $6 \%$ cross-linking is similar to that of the theoretical logarithmic spirals in Fig. 9, which have a fractal dimension of $d f \sim 1.85 \pm 0.15$.

Interestingly, the range of these fractal values is consistent with those observed in the literature for plastic shear bands [29]. It has also been observed that the fractal dimension depends on the dimensionless parameter $B$ in equation (7). The fractal dimension $d f$ is consistently larger in systems with lower $B$ values. This is observed in our experiments, if we assume the confining pressure and ratio of sound to boundary velocity to be constant for each gel. Hydrogels with higher percentages of monomer and cross-linking have a larger elastic modulus, and therefore a smaller $B$ and larger fractal dimension.

\section{Conclusions}

Polyacrylamide hydrogels were synthesized to study fluiddriven fractures in an impermeable elastic medium. These gels allow detailed measurements of the radial extent and full-field thickness of a fracture, as well as the fracture energy of the particular gel configuration. The transparent gels permit hydraulic fracturing experiments with a significant range of Young's modulus and fracture energy values. The sufficiently wide range of experimental parameters ensures both toughness and viscosity limiting regimes can be characterised fully in the context of dynamic hydraulic fracturing (see O'Keeffe et. al (2017), JFM, submitted).

This approach to studying hydraulic fracturing also gives rise to crack surface deformation patterns, which have been observed in fractured rock formations. The spacing between these phenomena is seen to depend heavily on the chemical configuration of the specific gel matrix as well as the fracture velocity. These step-lines patterns appear to occur due to minuscule inhomogeneities within the gel and/or small areas of the crack-tip experiencing mode III loading, due to crack tip softening and cavitation, that mimics the effects seen in ductile materials.

The calculation of fractal dimension values for the stepline patterns are also easily obtainable, which will allow comparisons with geological field observations. This study demonstrates how these gels can be made, their physical properties obtained and their suitability to further explore these fracturing phenomena in great detail.

Acknowledgements We would like to thank David Page-Croft and the technicians in the G.K. Batchelor lab for their help in setting up the experiment. We acknowledge helpful discussions in the initial stages of this project with Finn Box from the BP Institute, as well as Dominique Hoogland and Oren Scherman from the Department of Chemistry, University of Cambridge.

Open Access This article is distributed under the terms of the Creative Commons Attribution 4.0 International License (http://creativecommons.org/licenses/by/4.0/), which permits unrestricted use, distribution, and reproduction in any medium, provided you give appropriate credit to the original author(s) and the source, provide a link to the Creative Commons license, and indicate if changes were made.

\section{References}

1. Storm C, Pastore JJ, MacKintosh FC, Lubensky TC, Janmey PA (2005) Nonlinear elasticity in biological gels. Nature 435(7039): 191-194

2. Livne A, Cohen G, Ben-David O, Fineberg J (2004) Universal aspects of dynamic fracture in brittle materials. Experimental Chaos 742:122-131

3. Livne A, Cohen G, Fineberg J (2005) Universality and hysteretic dynamics in rapid fracture. Phys Rev Lett 94(22):224, 301

4. Livne A, Ben-David O, Fineberg J (2007) Oscillations in rapid fracture. Phys Rev Lett 98(12):124,301 
5. Goldman T, Harpaz R, Bouchbinder E, Fineberg J (2012) Intrinsic nonlinear scale governs oscillations in rapid fracture. Phys Rev Lett 108(10):104,303

6. Denisin AK, Pruitt BL (2016) Tuning the range of polyacrylamide gel stiffness for mechanobiology applications. ACS Appl Mater Interfaces 8(34):21,893-21,902

7. Economides MJ, Nolte KG (2000) Reservoir stimulation, vol 18. Wiley Chichester

8. Fairhurst C (1964) Measurement of in-situ rock stresses. With particular reference to hydraulic fracturing. Rock Mechanics, United States, $\mathrm{p} 2$

9. Rudnicki JW (2000) Geomechanics. Int J Solids Struct 37(1):349_ 358

10. Murphy HD, Tester JW, Grigsby CO, Potter RM (1981) Energy extraction from fractured geothermal reservoirs in lowpermeability crystalline rock. J Geophys Res Solid Earth 86(B8):7145-7158

11. Mair R, Hight D (1994) Compensation grouting. World Tunnelling and Subsurface Excavation 7(8)

12. Lister JR, Kerr RC (1991) Fluid-mechanical models of crack propagation and their application to magma transport in dykes. J Geophys Res Solid Earth 96(B6): 10,049-10,077

13. Kanninen MF, Popelar CL (1985) Advanced fracture mechanics. Oxford, UK, Oxford University Press

14. Savitski AA, Detournay E (2002) Propagation of a penny-shaped fluid-driven fracture in an impermeable rock: asymptotic solutions. Int J Solids Struct 39(26):6311-6337

15. Rice JR (1968) Mathematical analysis in the mechanics of fracture. Fracture: an advanced treatise $2: 191-311$

16. Johnson AW, Harley B (2011) Mechanobiology of cell-cell and cell-matrix interactions. Springer Science \& Business Media

17. Reinhart-King CA, Dembo M, Hammer DA (2003) Endothelial cell traction forces on RGD-derivatized polyacrylamide substrata. Langmuir 19(5):1573-1579

18. Zhang J, Daubert CR, Foegeding EA (2005) Characterization of polyacrylamide gels as an elastic model for food gels. Rheol Acta 44(6):622-630

19. Tanaka Y, Fukao K, Miyamoto Y (2000) Fracture energy of gels. Eur Phys J E 3(4):395-401

20. Baumberger T, Caroli C, Martina D (2006) Solvent control of crack dynamics in a reversible hydrogel. Nat Mater 5(7):552-555
21. White ML (1960) The permeability of an acrylamide polymer gel. J Phys Chem 64(10):1563-1565

22. Dalziel SB (2006) Digiflow user guide. DL Research Partners, Version 1

23. Boudou T, Ohayon J, Picart C, Tracqui P (2006) An extended relationship for the characterization of Young's modulus and Poisson's ratio of tunable polyacrylamide gels. Biorheology 43(6):721-728

24. Hertz H (1881) On the contact of elastic solids. J Reine Angew Math 92:156-171

25. MacKay JL, Kumar S (2013) Measuring the elastic properties of living cells with atomic force microscopy indentation. Methods Mol Biol 931:313-329

26. Bunger AP (2006) A photometry method for measuring the opening of fluid-filled fractures. Meas Sci Technol 17(12):3237

27. Tanaka Y, Fukao K, Miyamoto Y, Nakazawa H, Sekimoto K (1996) Regular patterns on fracture surfaces of polymer gels. J Phys Soc Jpn 65(8):2349-2352

28. Wallner H (1939) Linienstrukturen an bruchflächen. Zeitschrift für Physik 114(5-6):368-378

29. Poliakov ANB, Herrmann HJ, Podladchikov YY, Roux S (1994) Fractal plastic shear bands. Fractals 2(04):567-581

30. Narayan RL, Tandaiya P, Narasimhan R, Ramamurty U (2014) Wallner lines, crack velocity and mechanisms of crack nucleation and growth in a brittle bulk metallic glass. Acta Mater 80:407420

31. Xi XK, Zhao DQ, Pan MX, Wang WH, Wu Y, Lewandowski JJ (2006) Periodic corrugation on dynamic fracture surface in brittle bulk metallic glass. Appl Phys Lett 89(18):181,911

32. Sharon E, Cohen G, Fineberg J (2002) Crack front waves and the dynamics of a rapidly moving crack. Phys Rev Lett 88(8):85,503

33. Antoniou A, Bastawros A, Biner B (2007) Experimental observations of deformation behavior of bulk metallic glasses during wedge-like cylindrical indentation. J Mater Res 22(02):514 524

34. Jeong HY, Li XW, Yee AF, Pan J (1994) Slip lines in front of a round notch tip in a pressure-sensitive material. Mech Mater 19(1):29-38

35. Sarkar N, Chaudhuri BB (1994) An efficient differential boxcounting approach to compute fractal dimension of image. IEEE Trans Syst Man Cybern 24(1):115-120 